\title{
Review Paper: Association of Transforming Growth Factor Beta-1 -509C/T Gene Polymorphism with Ischemic Stroke: A Meta Analysis
}

\author{
Pradeep Kumar ${ }^{1}$, Amit Kumar', Mukesh Kumar Srivastava ${ }^{2}$, Shubham Misra ${ }^{1}$, Awadh Kishor Pandit ${ }^{1}$, Kameshwar Prasad $^{\text {* }}$
}

1. Department of Neurology, All India Institute of Medical Sciences, New Delhi, India.

2. Department of Neurobiochemistry, All India Institute of Medical Sciences, New Delhi, India.

Citation: Kumar, P., Kumar, A., Kumar Srivastava, M., Misra, Sh., Kishor Pandit, A., \& Prasad, K. (2016). Association of transforming growth factor beta-1 -509C/T gene polymorphism with ischemic stroke: A Meta Analysis. Basic and Clinical Neuroscience, 7(2), 91-96. http://dx.doi.org/10.15412/J.BCN.03070202

http://dx.doi.org/10.15412/J.BCN.03070202

Article info:

Received: 15 June 2015

First Revision: 13 July 2015

Accepted: 12 September 2015
Key Words:

Transforming growth factor beta, Cytokine, Inflammation, Cerebral infarction, Ischemic stroke, Single nucleotide polymorphism, Metaanalysis

\begin{abstract}
A B S T RA C T
Introduction: Transforming Growth Factor-Beta 1 (TGF- $\beta 1$ ) is a pleiotropic cytokine with potent anti-inflammatory property, which has been considered as an essential risk factor in the inflammatory process of Ischemic Stroke (IS), by involving in the pathophysiological progression of hypertension, atherosclerosis, and lipid metabolisms. -509C/T TGF- $\beta 1$ gene polymorphism has been found to be associated with the risk of IS. The aim of this meta-analysis was to provide a relatively comprehensive account of the relation between $-509 \mathrm{C} / \mathrm{T}$ gene polymorphisms of TGF- $\beta 1$ and susceptibility to IS.
\end{abstract}

Methods: A review of literature for eligible genetic association Studies published before October 20, 2014 was conducted in the PubMed, EMBASE, Google Scholar and Trip database. The strength of association was calculated by pooled odds ratios (ORs) with $95 \%$ confidence intervals using RevMan 5.3 software. Heterogeneity was examined using Higgins I-squared, Tau-squared, and Chi-squared tests.

Results: A total of 2 studies involving 614 cases and 617 controls were found. The overall estimates did not show any significant relation between TGF- $31-509 \mathrm{C} / \mathrm{T}$ polymorphism and risk of IS under dominant $(\mathrm{CC}+\mathrm{CT}$ vs. TT: $\mathrm{OR}=1.01,95 \% \mathrm{CI}=0.31$ to $3.26 ; \mathrm{P}=0.99)$, recessive $(\mathrm{CC}$ vs. $\mathrm{CT}+\mathrm{TT}$ : $\mathrm{OR}=0.94,95 \% \mathrm{CI}=0.47$ to $1.90 ; \mathrm{P}=0.87$ ), and allelic models ( $\mathrm{T}$ vs. $\mathrm{C}$ : $\mathrm{OR}=1.06$, $95 \% \mathrm{CI}=0.55$ to $2.04 ; \mathrm{P}=0.86$ ).

Conclusion: This meta-analysis showed that TGF- $\beta 1-509 \mathrm{C} / \mathrm{T}$ gene polymorphism has no significant association with the susceptibility of IS. Further well-designed prospective studies with larger sample size are needed to confirm these findings.

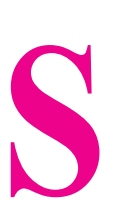

\section{Introduction}

troke is the third leading cause of death worldwide after Ischemic Heart Disease (IHD) and Cancer (Feigin et al., 2014).
Approximately $87 \%$ of stroke cases are ischemic in origin. Ischemic stroke (IS) is a complex multifactorial disease involving complex interactions between genetic and environmental factors (Dichgans, 2007). Emerging evidence has demonstrated that inflammation play an important role in

\section{* Corresponding Author:}

Kameshwar Prasad, DM

Address: Department of Neurology, All India Institute of Medical Sciences, New Delhi, India.

Tel: +91 (11) $26593497 \quad$ Fax: +91 (11) 26588663

E-mail: kp0704@gmail.com 
the pathogenesis of IS even though the exact mechanism is still unknown. As one of the candidate susceptible genes of IS, the functional polymorphism of inflammatory factors has become one of the most popular scientific investigations.

Transforming Growth Factor Beta-1 (TGF- $\beta 1$ ) is a pleiotropic cytokine with potent anti-inflammatory property, and has been considered an essential risk factor in the inflammatory process of IS by involving in the pathophysiological progression of lipid metabolisms, hypertension, and atherosclerosis (Libby, 2002; Chen, Liu, Fliesler, Han, Huang, \& Huang, 2007; Dobolyi, Vincze, Pál, \& Lovas, 2012; van der Spuy \& Pretorius, 2012; Lijnen, Petrov, \& Fagard, 2003). TGF- $\beta 1$ gene is located on chromosome 19 (q13.1-13.3), including 7 exons and 6 introns (Fujii, Brissenden, Derynck, \& Francke, 1986).

There are several common known single nucleotide polymorphisms (SNPs) in this gene. Among them, -509C/T (rs1800469) single nucleotide polymorphism (SNP) at position-509 relative to the first major transcription start site was found to be differentially related to transcription factor binding to the TGF- $\beta 1$ promoter, transcriptional activity of TGF- $\beta 1$, and TGF- $\beta 1$ plasma concentration (Grainger et al., 1999). So far, few studies have been performed to investigate the association of $-509 \mathrm{C} / \mathrm{T}$ polymorphism of TGF- $\beta 1$ gene with risk of IS (Peng, Zhan, Chen, \& Xu, 2011), (Sie et al., 2006; Tao et al., 2011). However, the results remain controversial. We performed this meta-analysis combining eligible published literature based on quantitative synthesis to derive a more convincing estimation for the association of $-509 \mathrm{C} / \mathrm{T}$ polymorphism with IS risk.

\section{Materials \& Methods}

\subsection{Identification of relevant studies}

A literature review for eligible genetic association studies published before October 20, 2014 was conducted in the PubMed, EMBASE, Google Scholar and Trip database. The following combinations of main keywords were used: "Transforming Growth Factor Beta 1 OR "TGF- $\beta 1$ "; "Ischemic stroke OR cerebral infarction OR "IS"" and "genetic polymorphism OR single nucleotide polymorphisms OR SNP". The search was done in any language, but only included those studies that were conducted on human subjects. All references in eligible articles were extensively reviewed to identify additional published articles.

\subsection{Inclusion and exclusion criteria}

To be included in the analysis, eligible studies had to meet the following criteria: 1) Studies on the association of $-509 \mathrm{C} / \mathrm{T}$ genetic polymorphisms of TGF- $\beta 1$ with susceptibility to IS; 2) All patients in the candidate studies meet the diagnostic criteria for IS through neuroimaging computer tomography (CT) or magnetic resonance imaging (MRI); 3) Studies with sufficient available genotypic data to calculate ORs with corresponding 95\%CI. The major reasons for excluding studies were as follows: 1) Not being case-control study; 2) Duplicate publications with overlapping subjects from the same study; and 3) No available data reported. This meta-analysis was conducted according to the Preferred Reporting Items for Systematic Reviews and Meta-analyses (PRISMA) guidance (Moher, Liberati, Tetzlaff, Altman, \& PRISMA Group, 2009).

\subsection{Data extraction}

According to the PRISMA guidance, two investigators (Pradeep Kumar and Amit Kumar) independently checked each full-text report for eligibility and extracted the following data from eligible studies: Surname of the first author, year of publication, country of origin, ethnicity, number of case and control subjects, age, sex ratio, genotyping method, allele and genotype frequency, etc. Disagreements were solved by discussion between all authors until consensus was reached.

\subsection{Quality assessment}

Newcastle-Ottawa Scale (NOS) criteria were used to assess the qualities of all included studies. The NOS criteria use a "star" rating system to judge methodological quality based on 3 aspects of a study: selection, comparability, and exposure. Scores range from 0 stars (worst) to 9 stars (best), with a score of 5 or higher indicating a moderate to high methodological quality. Two authors (Pradeep Kumar and Amit Kumar) independently assessed the quality of included studies. Discrepancies over quality scores were resolved by discussion with all authors and subsequent consensus.

\subsection{Statistical analysis}

The control group was tested by Hardy Weinberg Equilibrium (HWE) to compare the expected and observed genotype frequencies using the Pearson and Chi-square tests for goodness of fit. The relation between the TGF- $\beta 1$ 
genetic polymorphisms and susceptibility to IS was assessed by the pooled odds ratios (ORs) with their corresponding 95\% confidence intervals under 3 genetic models of dominant, recessive, and allelic one (using RevMan 5.3 software). Taking into consideration the possible betweenstudy heterogeneity, a statistical test for that was first conducted using Cochran's Q statistic and the $\mathrm{I}^{2}$ metric (Attia, Thakkinstian, \& D'Este, 2003). A random-effects model was used to estimate the pooled ORs and 95\%CIs, as heterogeneity was found with $\mathrm{P}<0.10$ or $\mathrm{I}^{2}>50 \%$.

\section{Results}

A total of 64 relevant papers were identified using the prespecified search strategy. Figure 1 presents a flow diagram of retrieved and excluded studies with their reasons for exclusion. In accordance with the inclusion criteria, two studies were included in this meta-analysis; with a total of 614 IS patients and 617 controls. Studies were conducted in only one major Asian ethnic population. The publication years of included studies ranged from 2010 to 2011. All two studies had controls in HWE. The quality scores of all included studies were moderately high, with NOS stars more than 5 . The characteristics and methodological quality of these studies are summarized in Table 1.

\subsection{Association of TGF- $\beta 1-509 \mathrm{C} / \mathrm{T}$ polymorphism} with susceptibility to IS

The overall estimates did not show any significant relation between TGF- $\beta 1-509 \mathrm{C} / \mathrm{T}$ polymorphism and

Table 1. Characteristic of studies included in the meta-analysis of -509C/T TGF- $\beta 1$ gene -509C/T polymorphism association with the risk of ischemic stroke.

\begin{tabular}{|c|c|c|c|c|c|c|c|c|c|c|c|c|}
\hline S.No & Year & Author & Origin & Ethnicity & $\begin{array}{l}\text { Cases/con- } \\
\text { trols }\end{array}$ & HWE & $\begin{array}{l}\text { Matching } \\
\text { Criteria }\end{array}$ & $\begin{array}{c}\text { Genotyping } \\
\text { Method }\end{array}$ & $M / F$ & $\begin{array}{l}\text { Age, Mean } \\
\pm S . D\end{array}$ & $\begin{array}{l}\text { NOS } \\
\text { Star }\end{array}$ & $\begin{array}{c}\text { Source } \\
\text { of con- } \\
\text { trol }\end{array}$ \\
\hline \multirow{2}{*}{1.} & \multirow{2}{*}{2010} & \multirow{2}{*}{$\begin{array}{c}\text { Tao et al. (Tao } \\
\text { et al., 2011) }\end{array}$} & \multirow{2}{*}{ China } & \multirow{2}{*}{ Asian } & \multirow{2}{*}{$450 / 450$} & \multirow{2}{*}{ Yes } & \multirow{2}{*}{ NA } & \multirow{2}{*}{ ARMS-PCR } & $306 / 144$ & $64.87 \pm 14.4$ & \multirow{2}{*}{7.9} & \multirow{2}{*}{ PB } \\
\hline & & & & & & & & & $326 / 124$ & $63.85 \pm 13.8$ & & \\
\hline \multirow{2}{*}{2.} & \multirow{2}{*}{2011} & \multirow{2}{*}{$\begin{array}{l}\text { Peng et al. } \\
\text { (Peng et al., } \\
\text { 2011) }\end{array}$} & \multirow{2}{*}{ China } & \multirow{2}{*}{ Asian } & \multirow{2}{*}{$164 / 167$} & \multirow{2}{*}{ Yes } & \multirow{2}{*}{ Age+Sex } & \multirow{2}{*}{ PCR-RFLP } & $100 / 64$ & $63.68 \pm 6.65$ & \multirow{2}{*}{7.9} & \multirow{2}{*}{ PB } \\
\hline & & & & & & & & & $95 / 72$ & $64.87 \pm 6.16$ & & \\
\hline
\end{tabular}

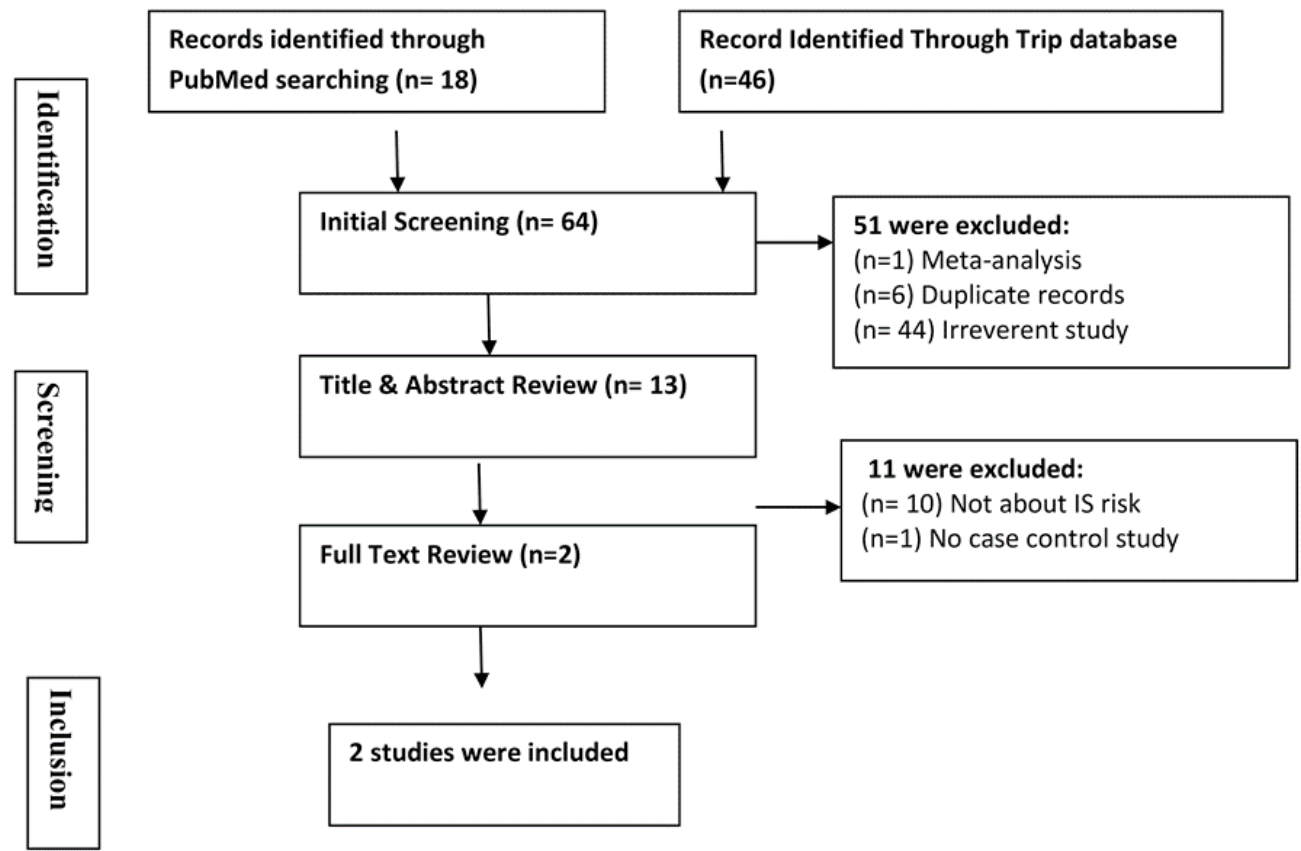

Figure 1. Flow diagram of the selection of studies and reasons for exclusion from the present meta-analysis. 


\section{A. Dominant Model TGF -509C/T}

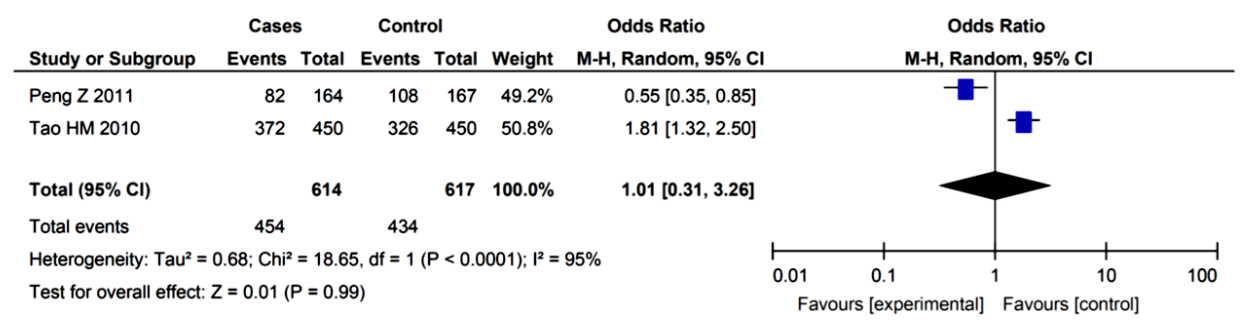

\section{B. Recessive Model TGF -509C/T}

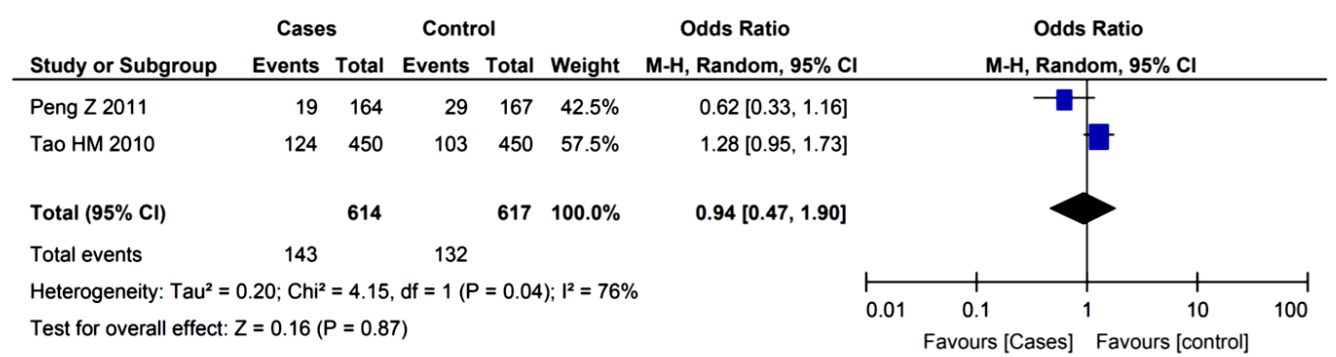

\section{Allelic Model T vs. C TGF -509C/T}

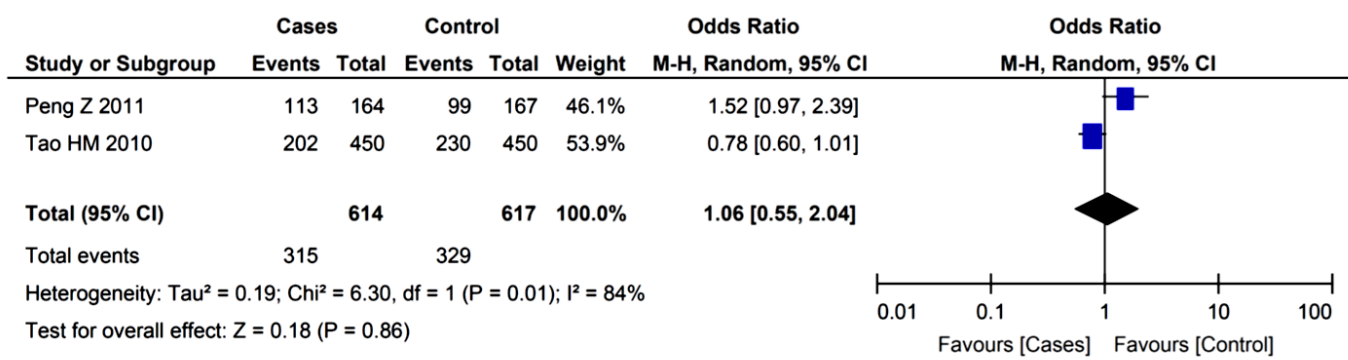

NEUR:SCIENCE

Figure 4. Forest plots of ORs for the association of the TGF -509C/T polymorphism and susceptibility to Ischemic stroke, (A) Dominant model (CC+CT vs. TT), (B) Recessive Model (CC vs. CT+TT), (C) Allelic Model (T allele vs. C allele).

risk of IS under dominant $(\mathrm{CC}+\mathrm{CT}$ vs. TT: $\mathrm{OR}=1.01$, $95 \% \mathrm{CI}=0.31$ to $3.26 ; \mathrm{P}=0.99$ ), recessive ( $\mathrm{CC}$ vs. $\mathrm{CT}+\mathrm{TT}$ : $\mathrm{OR}=0.94,95 \% \mathrm{CI}=0.47$ to $1.90 ; \mathrm{P}=0.87$ ), and allelic models ( $\mathrm{T}$ vs. $\mathrm{C}$ : $\mathrm{OR}=1.06,95 \% \mathrm{CI}=0.55$ to $2.04 ; \mathrm{P}=0.86$ ) indicating that $\mathrm{CT}$ and $\mathrm{TT}$ genotypes may not possibly confer an increased susceptibility to IS compared to CC genotype (Figure 2).

\section{Discussion}

Ischemic stroke is a multifactorial disease significantly associated with certain genetic factors. Many genome association and candidate gene studies have been conducted to identify IS susceptible genes; discovering novel molecular pathways and helping susceptible individuals prevent the development of IS. Evidence suggests that inflammation and IS are interrelated. TGF- $\beta 1$ is a pleiotropic anti-inflammatory cytokine involving in numer- 
ous inflammatory processes as well as the development of IS. The protein expression of TGF- $\beta 1$ is predominantly under genetic control of TGF- $\beta 1$ gene. Various common SNPs in this gene can modify the protein expression of TGF- $\beta 1$ and $-509 \mathrm{C} / \mathrm{T}$ genetic polymorphism of TGF- $\beta 1$ has been implicated to be associated with increased serum TGF- $\beta 1$ level. Only 3 epidemiological studies investigated the association of $-509 \mathrm{C} / \mathrm{T}$ polymorphism with susceptibility to IS, yet with conflicting results. Two studies included were of case control design and one study conducted by Sie et al. was excluded due to cohort study design (Sie et al., 2006).

Therefore, we conducted this meta-analysis by combining only 2 eligible published studies to draw a more precise conclusion. Under all genetic models, overall estimates did not observe any significant relation between TGF- $\beta 1-509 \mathrm{C} / \mathrm{T}$ polymorphism and risk of IS.

We were unable to draw a solid conclusion for the association between $-509 \mathrm{C} / \mathrm{T}$ polymorphism and the risk of IS. Among all genetic models, there was some evidence of heterogeneities between the included studies. The present study is the first meta-analysis to our knowledge, concerning relation between TGF- $\beta 1$ gene $-509 \mathrm{C} / \mathrm{T}$ polymorphism and risk of IS. The genotypic frequencies in control groups of the included studies were in accordance to HWE, demonstrating the true representation of control samples. The methodological quality of the included studies was of good quality as assessed by NOS quality score.

Limitations of our current study were as follows: first, combined sample size of the included studies were still inadequate to detect the association of $-509 \mathrm{C} / \mathrm{T}$ polymorphism with IS risk. Second, we only focused on one variation in TGF- $\beta 1$ gene, without considering the role of other SNPs in this gene or precluding the potential interactions such as linkage disequilibrium or haplotype analysis between them, which might mask its real genetic effect towards IS susceptibility. Third, IS is a complex multifactorial disease related to both genetic and environmental factors, such as climate, diet, life style and economic status, etc. However, our results were based on unadjusted estimates as most of original studies did not take such gene-environmental interactions into account.

Well-designed prospective studies are needed to reduce the potential sources of biasing by including clinical characteristics such as smoking, alcohol drinking, family history of stroke, diabetes mellitus, hypertension, dyslipidemia, cardiac diseases as well as matching criteria of cases and control, subtypes of IS, and use of standard genotyping methods.

In our meta-analysis we did not find any significant association between two included with single gene polymorphism and IS. However due to heterogeneity of the studies, it is too inconsistent to draw conclusion for the association of these gene polymorphism with IS susceptibility. More convincing evidence is required to draw conclusion. Therefore, well-designed studies are needed to investigate the association of these polymorphisms in larger and different ancestry populations.

\section{References}

Attia, J., Thakkinstian, A., \& D'Este, C. (2003). Meta-analyses of molecular association studies: methodologic lessons for genetic epidemiology. Journal of Clinical Epidemiology, 56(4), 297-303.

Chen, C. L., Liu, I. H., Fliesler, S. J., Han, X., Huang, S. S., \& Huang, J. S. (2007). Cholesterol suppresses cellular TGF-beta responsiveness: Implications in atherogenesis. Journal of Cell Science, 120(Pt 20), 3509-3521. doi: 10.1242/jcs.006916.

Dichgans, M. (2007). Genetics of ischaemic stroke. Lancet Neurology, 6(2), 149-161. doi: 10.1016/S1474-4422(07)70028-5.

Dobolyi, A., Vincze, C., Pál, G., \& Lovas, G. (2012). The neuroprotective functions of transforming growth factor Beta proteins. International Journal of Molecular Sciences, 13(7), 8219-8258. doi: 10.3390/ijms13078219.

Feigin, V. L., Forouzanfar, M. H., Krishnamurthi, R., Mensah, G. A., Connor, M., Bennett, D. A., et al. (2014). Global and regional burden of stroke during 1990-2010: Findings from the Global Burden of Disease Study 2010. Lancet, 383(9913), 245-254.

Fujii, D., Brissenden, J. E., Derynck, R., \& Francke, U. (1986). Transforming growth factor beta gene maps to human chromosome 19 long arm and to mouse chromosome 7. Somatic Cell and Molecular Genetics, 12(3), 281-288.

Grainger, D. J., Heathcote, K., Chiano, M., Snieder, H., Kemp, P R., Metcalfe, J. C., et al. (1999). Genetic control of the circulating concentration of transforming growth factor type beta1. Human Molecular Genetics, 8(1), 93-97.

Libby, P. (2002). Inflammation in atherosclerosis. Nature, 420(6917), 868-874. doi: 10.1038/nature01323

Lijnen, P. J., Petrov, V. V., \& Fagard, R. H. (2003). Association between transforming growth factor-beta and hypertension. American Journal of Hypertension, 16(7), 604-611.

Moher, D., Liberati, A., Tetzlaff, J., Altman, D. G., \& PRISMA Group. (2009). Preferred reporting items for systematic reviews and meta-analyses: The PRISMA statement. Annals of internal medicine, 151(4), 264-269.

Peng, Z., Zhan, L., Chen, S., \& Xu, E. (2011). Association of transforming growth factor- $\beta 1$ gene C-509T and T869C polymorphisms with atherosclerotic cerebral infarction in the Chinese: 
a case-control study. Lipids in Health and Disease, 10(1), 100-107. doi: 10.1186/1476-511X-10-100.

Sie, M. P. S., Uitterlinden, A. G., Bos, M. J., Arp, P. P., Breteler, M. M. B., Koudstaal, P. J., et al. (2006). TGF-beta 1 polymorphisms and risk of myocardial infarction and stroke: the Rotterdam Study. Stroke; A Journal of Cerebral Circulation, 37(11), 2667-2671. doi: 10.1161/01.STR.0000244779.30070.1a.

Tao, H., Chen, G., Lu, X., Hu, X., Chen, G., \& Shao, B. (2011). TGF-beta 1 codon 10 polymorphism is associated with cerebral SVD. The Canadian Journal of Neurological Sciences. Le Journal Canadien Des Sciences Neurologiques, 38(6), 869-873.

Van der Spuy, W. J., \& Pretorius, E. (2012). Interrelation between inflammation, thrombosis, and neuroprotection in cerebral ischemia. Reviews in the Neurosciences, 23(3), 269-278. doi: 10.1515/revneuro-2012-0028. 